\title{
Complex dynamics of a discrete predator-prey system with a strong Allee effect on the prey and a ratio-dependent functional response
}

Qiquan Fang ${ }^{1}$ and Xianyi Li ${ }^{1 *}$ (D)

"Correspondence:

mathxyli@zust.edu.cn

'School of Science, Zhejiang

University of Science and

Technology, Hangzhou, P.R. China

\section{Springer}

\begin{abstract}
In this paper, we consider the complex dynamics of a discrete predator-prey system with a strong Allee effect on the prey and a ratio-dependent functional response, which is the discrete version of the continuous system in (Nonlinear Anal., Real World Appl. 16:235-249, 2014). First, by giving several examples to display the limitations and errors of the local stability of the equilibrium point $\mathbf{p}$ obtained in (Nonlinear Anal., Real World Appl. 16:235-249, 2014), we provide an easily verified and complete discrimination criterion for the local stability of this equilibrium. Then we study some properties of its discrete version, especially for the stability and bifurcation for the equilibrium point $E_{1}$, which has not been considered in any literature to the best of our knowledge. By using the center manifold theorem and bifurcation theory, we consider the flip bifurcation of this system at $E_{1}$ and obtain the stability of the closed orbits bifurcated from $E_{1}$. The numerical simulations not only show the correctness of our theoretical analysis, but also we find some new and interesting dynamics of this system.
\end{abstract}

Keywords: Discrete predator-prey model; Strong Allee effect; Flip bifurcation; Center Manifold Theorem

\section{Introduction}

It is well known that one of the most challenging investigation areas for the biology and/or ecology population is the predator-prey interaction among the population. In the past few decades, many ecologists, mathematicians and biologists have paid attention to this field, especially for the persistence and/or extinction of one or more predator-predator interacting groups $[8,9,23]$.

From the modeling point of view, a potential mechanism that makes possible mutual extinction and creates long-term oscillation behavior is the interaction of predators and prey, including ratio-dependent functional responses $[3,4,11,25]$. With such a ratio-dependent method, the growth rate of per capita predator is a function of the ratio of prey to predator abundance.

In 1931, Allee pointed out that when the population size gets larger, the per capita growth rates will start to rise and then fall. We call such a biological phenomenon an Allee

(c) The Author(s) 2018. This article is distributed under the terms of the Creative Commons Attribution 4.0 International License (http://creativecommons.org/licenses/by/4.0/), which permits unrestricted use, distribution, and reproduction in any medium, provided you give appropriate credit to the original author(s) and the source, provide a link to the Creative Commons license, and indicate if changes were made. 
effect; it is characterized by the positive correlation between the population size and the average individual fitness of the population. There are two main types of Allee effect, which are known as the strong Allee effect and the weak Allee effect. The strong Allee effect indicates that there exists a critical population size under which the population growth rate becomes negative. The weak Allee effect means a reduced per capita growth rate at low population size but never becoming negative. There are many factors that lead to the Allee effect, such as difficulties in finding mates, social dysfunction and inbreeding depression. The Allee effect has also been found in wild ecosystems [5] and marine ecosystems [10].

There are many investigations for predator-prey models [2-12] and [23, 25]. In recent years, the discrete-time population models have attracted more and more attention. On the one hand, when the populations are not overlapping or the populations are small, the discrete-time models are more suitable than the continuous-time models. On the other hand, one can get more accurate numerical simulation results from discrete-time models. Moreover, the numerical simulation results are generally obtained by discretizing the corresponding continuous-time model. The discrete-time models sometimes have richer dynamical behaviors. For instance, the single-species discrete-time models have bifurcations, chaos and more complex dynamical behaviors (see [6, 12-16, 18-22]). For the flip bifurcation and Hopf bifurcation of discrete models, see also [6, 12-14, 24].

Recently, Aguirre et al. [1] considered the following continuous-time predator-prey system:

$$
\left\{\begin{array}{l}
\frac{d x}{d t}=x(1-x)(x-m)(x+y)-\alpha x y \\
\frac{d y}{d t}=\beta x y-\gamma y(x+y)
\end{array}\right.
$$

where the positive parameter $\gamma$ represents the death rate of the predator $y$; the parameter $\alpha>0$ is the largest prey mortality rate due to predation for an infinite number of predators, which is known as consumption capacity; the parameter $\beta>0$ is the maximum predator growth rate due to an infinite number of prey, which is called the predator growing capacity; and the parameter $m(0<m \ll 1)$ is a rescaled Allee threshold for the prey without predator.

Considering the biological significance of the system (1.1), one only takes into account the stability and bifurcation of its nonnegative equilibria.

For the sake of illustration, some denotations in [1] are still adopted as follows:

$$
\begin{aligned}
& \Delta=\Delta(\alpha, \beta, \gamma, m):=\beta(m-1)^{2}-4 \alpha(\beta-\gamma), \\
& H=H(\alpha, \beta, \gamma, m):=\beta \sqrt{\Delta}[(1+m) \sqrt{\beta}-\sqrt{\Delta}]-2 \gamma(\beta-\gamma)(\beta-\alpha), \\
& \mathbf{q}:=\left(\frac{m+1}{2}, \frac{(\beta-\gamma)(m+1)}{2 \gamma}\right), \\
& \mathbf{p}_{\mathbf{s}}:=\left(\frac{\beta(m+1)-\sqrt{\beta \Delta}}{2 \beta}, \frac{(\beta-\gamma)[\beta(m+1)-\sqrt{\beta \Delta}]}{2 \beta \gamma}\right),
\end{aligned}
$$

and

$$
\mathbf{p}:=\left(\frac{\beta(m+1)+\sqrt{\beta \Delta}}{2 \beta}, \frac{(\beta-\gamma)[\beta(m+1)+\sqrt{\beta \Delta}]}{2 \beta \gamma}\right) .
$$


We also define the following function used later:

$$
L=L(\alpha, \beta, \gamma, m):=\beta \sqrt{\Delta}[(1+m) \sqrt{\beta}+\sqrt{\Delta}]+2 \gamma(\beta-\gamma)(\beta-\alpha) .
$$

First of all, we notice that there are some obvious errors in [1] although there also are some good results in [1]. Let us view the following proposition (Theorem 4.2 in [1]).

Proposition A Let $\beta>\gamma$ and $\Delta>0$, then:

(a) the equilibrium point $\mathbf{p}_{\mathbf{s}}$ in system (1.1) is a hyperbolic saddle;

(b) if $H<0$, the equilibrium point $\mathbf{p}$ in system (1.1) is a hyperbolic attractor;

(c) if $H>0$, the equilibrium point $\mathbf{p}$ in system (1.1) is a hyperbolic repeller;

(d) if $H=0$, the equilibrium point $\mathbf{p}$ in system (1.1) undergoes a Hopf bifurcation.

The following counterexample indicates that the conclusion (c) in Proposition A is wrong.

Counterexample 1.1 Consider system (1.1) with the following parameters:

$$
m=0.1, \quad \alpha=2, \quad \beta=2, \quad \gamma=1.8 .
$$

According to the symbols in [1], one can easily obtain $\Delta=1 / 50, H=2 / 5>0$, and the equilibrium point $\mathbf{p}=(3 / 5,1 / 15)$. Thus the Jacobian matrix J of system (1.1) at the equilibrium point $\mathbf{p}$ is given by

$$
J_{\mathbf{p}}=\left(\begin{array}{cc}
2 / 25 & -27 / 25 \\
1 / 75 & -3 / 25
\end{array}\right),
$$

whose two eigenvalues are $-0.02 \pm 0.0663 i$. So, the equilibrium point $\mathbf{p}$ is a hyperbolic attractor. Therefore, the result (c) in Proposition A is not appropriate for this counterexample.

We now provide right results to correct Proposition A as follows.

Theorem 1.1 Let $\beta>\gamma$ and $\Delta>0$, then

(a) the equilibrium point $\mathbf{p}_{\mathbf{s}}$ is a hyperbolic saddle;

(b) if $L>0$, the equilibrium point $\mathbf{p}$ is a hyperbolic attractor;

(c) if $L<0$, the equilibrium point $\mathbf{p}$ is a hyperbolic repeller;

(d) if $L=0$, system (1.1) undergoes a Hopf bifurcation at the equilibrium point $\mathbf{p}$.

Proof We just prove the parts (b), (c) and (d), since the proof of part (a) is the same as in [1].

Parts (b) and (c). By some simple computations, we give the determinant of the Jacobian matrix $J_{\mathbf{p}}$ by

$$
\operatorname{Det}\left(J_{\mathbf{p}}\right)=\frac{\beta-\gamma}{8 \beta \gamma} \sqrt{\Delta}[(1+m) \sqrt{\beta}+\sqrt{\Delta}]^{3}>0
$$


Hence, the local stability of the equilibrium $\mathbf{p}$ is determined by the sign of the trace of the Jacobian matrix $J_{\mathbf{p}}$. Note that

$$
\operatorname{Tr}\left(J_{\mathbf{p}}\right)=-\frac{(1+m) \sqrt{\beta}+\sqrt{\Delta}}{4 \beta^{\frac{3}{2}} \gamma} L
$$

we have $\operatorname{sign}\left(\operatorname{Tr}\left(J_{\mathbf{p}}\right)\right)=-\operatorname{sign}(L)$. Therefore, by the Hartman-Grobman theorem [7, 17], the parts (b) and (c) hold.

Part (d). Let $L=0$, then $\operatorname{Tr}\left(J_{\mathbf{p}}\right)=0$, and therefore the eigenvalues of the Jacobian matrix $J_{\mathbf{p}}$ are $\pm \frac{\beta-\gamma}{2 \beta} \sqrt{\alpha-\beta} i$, which cross the imaginary axis. Hence, system (1.1) undergoes a Hopf bifurcation at the equilibrium point $\mathbf{p}$.

Remark 1.1 Comparing Theorem 1.1 with Proposition A, one can find that the part (b) in Proposition A is only partly true. In fact, for $\beta>\gamma$, if $H<0$, i.e.,

$$
0<\beta \sqrt{\Delta}[(1+m) \sqrt{\beta}-\sqrt{\Delta}]<2 \gamma(\beta-\gamma)(\beta-\alpha)
$$

then $\beta>\alpha$. Hence, $L=\beta \sqrt{\Delta}[(1+m) \sqrt{\beta}+\sqrt{\Delta}]+2 \gamma(\beta-\gamma)(\beta-\alpha)>0$. By Theorem 1.1, the part (b) in Proposition A holds.

Remark 1.2 Also, one can find that the part (d) in Proposition A is wrong. In fact, for $\beta>\gamma$, if $H=0$, i.e.,

$$
\beta \sqrt{\Delta}[(1+m) \sqrt{\beta}-\sqrt{\Delta}]=2 \gamma(\beta-\gamma)(\beta-\alpha)
$$

then

$$
\operatorname{Tr}\left(J_{\mathbf{p}}\right)=-\frac{\alpha(m+1) \sqrt{\Delta}}{4 \gamma}[(1+m) \sqrt{\beta}+\sqrt{\Delta}]<0 .
$$

Hence, the equilibrium point $\mathbf{p}$ in system (1.1) cannot undergo a Hopf bifurcation.

Next, we consider the following discrete version of system (1.1) with strong Allee effect:

$$
\left\{\begin{array}{l}
x(n+1)=x(n) \exp [(1-x(n))(x(n)-m)(x(n)+y(n))-\alpha y(n)], \\
y(n+1)=y(n) \exp [\beta x(n)-\gamma(x(n)+y(n))]
\end{array}\right.
$$

where the positive parameters $m, \alpha, \beta, \gamma$ and their biological significance are the same as in system (1.1). If the initial values of system (1.5) are positive, one can prove that the corresponding solutions of system (1.5) $(x(n), y(n))$ are also positive.

The rest of this paper is organized as follows. In Sect. 2, we consider the existence and local stability of equilibria of system (1.5). In Sect. 3, we give the flip and fold bifurcations for the equilibrium point $E_{1}(1,0)$ by using center manifold theory. In Sect. 4 , we present the numerical simulations, which not only show the consistency between our results and theoretical analysis, but also we exhibit some complex dynamical behaviors, such as the cascade of period-doubling bifurcation in periods 2, 4 and 8, quasi-periodic orbits and chaotic sets. At last, some conclusions are presented in Sect. 5. 


\section{Equilibria and their dynamics}

In this section, we first determine the existence of the equilibria of system (1.5), then we investigate their dynamics.

\subsection{Existence of equilibria}

From system (1.5), we know the equilibria of system (1.5) satisfy the following equations:

$$
\left\{\begin{array}{l}
x=x \exp [(1-x)(x-m)(x+y)-\alpha y] \\
y=y \exp [\beta x-\gamma(x+y)]
\end{array}\right.
$$

In view of the biological meanings of system (1.5), one only needs to consider the existence of nonnegative equilibria. The following results are easy to deduce.

Theorem 2.1 System (1.5) always has boundary equilibrium points $E_{0}(0,0), E_{1}(1,0)$ and $E_{m}(m, 0)$. For the nonnegative equilibrium point $(s)$, the following statements hold.

(i) For $\beta>\gamma$,

(i.1) if $\Delta>0$, then $\mathbf{p}_{\mathbf{s}}$ and $\mathbf{p}$ are two distinct positive equilibria of system (1.5);

(i.2) if $\Delta=0$, then $\mathbf{p}_{\mathbf{s}}$ and $\mathbf{p}$ collide into a unique positive equilibrium $\mathbf{q}$.

(ii) For $\beta=\gamma, \mathbf{p}$ and $\mathbf{p}_{\mathbf{s}}$ become into the equilibria $E_{1}$ and $E_{m}$, respectively.

In order to investigate the local stability and bifurcation for an equilibrium point of a general $2 D$ system, the following lemma will be very useful and even essential; for the details see [24].

Lemma 2.1 Let $F(\lambda)=\lambda^{2}+B \lambda+C$, where $B$ and $C$ are two real constants. Suppose $\lambda_{1}$ and $\lambda_{2}$ are two roots of $F(\lambda)=0$. Then the following statements hold.

(i) If $F(1)>0$, then

(i.1) $\left|\lambda_{1}\right|<1$ and $\left|\lambda_{2}\right|<1$ if and only if $F(-1)>0$ and $C<1$;

(i.2) $\lambda_{1}=-1$ and $\lambda_{2} \neq-1$ if and only if $F(-1)=0$ and $B \neq 2$;

(i.3) $\left|\lambda_{1}\right|<1$ and $\left|\lambda_{2}\right|>1$ if and only if $F(-1)<0$;

(i.4) $\left|\lambda_{1}\right|>1$ and $\left|\lambda_{2}\right|>1$ if and only if $F(-1)>0$ and $C>1$;

(i.5) $\lambda_{1}$ and $\lambda_{2}$ are a pair of conjugate complex roots with $\left|\lambda_{1}\right|=\left|\lambda_{2}\right|=1$ if and only if $-2<B<2$ and $C=1$;

(i.6) $\lambda_{1}=\lambda_{2}=-1$ if and only if $F(-1)=0$ and $B=2$.

(ii) If $F(1)=0$, namely, 1 is one root of $F(\lambda)=0$, then the other root $\lambda$ satisfies

$|\lambda|=(<,>) 1$ if and only if $|C|=(<,>) 1$.

(iii) If $F(1)<0$, then $F(\lambda)=0$ has one root lying in $(1, \infty)$. Moreover,

(iii.1) the other root $\lambda$ satisfies $\lambda<(=)-1$ if and only if $F(-1)<(=) 0$;

(iii.2) the other root $\lambda$ satisfies $-1<\lambda<1$ if and only if $F(-1)>0$.

Now, let us recall the definition of topological types for an equilibrium point $(\bar{x}, \bar{y})$.

Definition 2.1 Let $\bar{E}(\bar{x}, \bar{y})$ be an equilibrium point of system (1.5) with multipliers $\lambda_{1}$ and $\lambda_{2}$.

(i) The point $\bar{E}(\bar{x}, \bar{y})$ is called sink if $\left|\lambda_{1}\right|<1$ and $\left|\lambda_{2}\right|<1$, so sink is locally asymptotically stable. 
(ii) The point $\bar{E}(\bar{x}, \bar{y})$ is called source if $\left|\lambda_{1}\right|>1$ and $\left|\lambda_{2}\right|>1$, so source is locally asymptotically unstable.

(iii) The point $\bar{E}(\bar{x}, \bar{y})$ is called saddle if $\left|\lambda_{1}\right|<1$ and $\left|\lambda_{2}\right|>1$ (or $\left|\lambda_{1}\right|>1$ and $\left|\lambda_{2}\right|<1$ ).

(iv) The point $\bar{E}(\bar{x}, \bar{y})$ is called non-hyperbolic if either $\left|\lambda_{1}\right|=1$ or $\left|\lambda_{2}\right|=1$.

\subsection{The dynamics of boundary equilibria}

In this subsection, we discuss the local dynamics for the boundary equilibria of system (1.5). By some computations, one can easily derive the following results.

\section{Theorem 2.2}

(i) The equilibrium point $E_{0}$ is always non-hyperbolic;

(ii) if $\beta<\gamma$, then the equilibrium point $E_{1}$ is a sink, but equilibrium $E_{m}$ is a saddle;

(iii) if $\beta=\gamma$, then the equilibria $E_{1}$ and $E_{m}$ are non-hyperbolic;

(iv) if $\beta>\gamma$, then the equilibrium point $E_{1}$ is a saddle, while the equilibrium $E_{m}$ is a source.

\subsection{The dynamics of positive equilibria}

Theorem 2.3 Assume that $\beta>\gamma$ and $\Delta=0$, then the positive equilibrium point $\mathbf{q}$ is nonhyperbolic.

Proof The Jacobian matrix J of system (1.5) at the point $\mathbf{q}$ is

$$
J_{\mathbf{q}}=\left(\begin{array}{cc}
1+\frac{1}{8}(m+1)(m-1)^{2} & \frac{1}{8}(m+1)(m-1)^{2}-\frac{1}{2} \alpha(m+1) \\
\frac{(\beta-\gamma)^{2}(m+1)}{2 \gamma} & 1-\frac{1}{2}(\beta-\gamma)(m+1)
\end{array}\right) .
$$

The characteristic equation associated with $(2.2)$ is

$$
F(\lambda):=\lambda^{2}-\operatorname{Tr}\left(J_{\mathbf{q}}\right) \lambda+\operatorname{Det}\left(J_{\mathbf{q}}\right)=0,
$$

where $\operatorname{Tr}\left(J_{\mathbf{q}}\right)$ and $\operatorname{Det}\left(J_{\mathbf{q}}\right)$ are the trace and determinant of the matrix $J_{\mathbf{q}}$ respectively, namely,

$$
\operatorname{Tr}\left(J_{\mathbf{q}}\right)=2+\frac{1}{8 \alpha}(\alpha-\beta)(m+1)(m-1)^{2}
$$

and

$$
\operatorname{Det}\left(J_{\mathbf{q}}\right)=1+\frac{1}{8 \alpha}(\alpha-\beta)(m+1)(m-1)^{2} .
$$

Through some computations, we can see that its two roots are 1 and $1+\frac{1}{8 \alpha}(\alpha-\beta)(m+$ $1)(m-1)^{2}$. Therefore, the positive equilibrium point $\mathbf{q}$ is non-hyperbolic.

We are now in a position to consider the stability of positive equilibria $\mathbf{p}_{\mathbf{s}}$ and $\mathbf{p}$.

Theorem 2.4 Let $\beta>\gamma$ and $\Delta>0$, then the following statements hold.

(a) If $\frac{\beta-\gamma}{8 \beta \gamma} \sqrt{\Delta}[(1+m) \sqrt{\beta}-\sqrt{\Delta}]^{3}-\frac{(1+m) \sqrt{\beta}-\sqrt{\Delta}}{2 \beta^{\frac{3}{2}} \gamma} H>4$, then the point $\mathbf{p}_{\mathbf{s}}$ is a source; 
(b) if $\frac{\beta-\gamma}{8 \beta \gamma} \sqrt{\Delta}[(1+m) \sqrt{\beta}-\sqrt{\Delta}]^{3}-\frac{(1+m) \sqrt{\beta}-\sqrt{\Delta}}{2 \beta^{\frac{3}{2}} \gamma} H=4$, then the point $\mathbf{p}_{\mathbf{s}}$ is non-hyperbolic;

(c) if $\frac{\beta-\gamma}{8 \beta \gamma} \sqrt{\Delta}[(1+m) \sqrt{\beta}-\sqrt{\Delta}]^{3}-\frac{(1+m) \sqrt{\beta}-\sqrt{\Delta}}{2 \beta^{\frac{3}{2}} \gamma} H<4$, then the point $\mathbf{p}_{\mathbf{s}}$ is a saddle.

Proof The Jacobian matrix $J$ of system (1.5) at the equilibrium point $\mathbf{p}_{\mathbf{s}}\left(x_{1}, y_{1}\right)$ is

$$
J_{\mathbf{p}_{\mathbf{s}}}=\left(\begin{array}{cc}
1+x_{1}\left[\alpha\left(1-\frac{\gamma}{\beta}\right)+\frac{\sqrt{\beta \Delta}}{\gamma} x_{1}\right] & -\frac{\alpha \gamma}{\beta} x_{1} \\
\frac{(\beta-\gamma)^{2}}{\gamma} x_{1} & 1-(\beta-\gamma) x_{1}
\end{array}\right) .
$$

Its characteristic equation is

$$
F(\lambda)=\lambda^{2}-\operatorname{Tr}\left(J_{\mathbf{p s}}\right) \lambda+\operatorname{Det}\left(J_{\mathbf{p}_{\mathbf{s}}}\right)=0,
$$

where

$$
\operatorname{Tr}\left(J_{\mathbf{p s}_{\mathbf{s}}}\right)=2+\frac{(1+m) \sqrt{\beta}-\sqrt{\Delta}}{4 \beta^{\frac{3}{2}} \gamma} H
$$

and

$$
\operatorname{Det}\left(J_{\mathbf{p}_{\mathbf{s}}}\right)=1+\frac{(1+m) \sqrt{\beta}-\sqrt{\Delta}}{4 \beta^{\frac{3}{2}} \gamma} H-\frac{\beta-\gamma}{8 \beta \gamma} \sqrt{\Delta}[(1+m) \sqrt{\beta}-\sqrt{\Delta}]^{3} .
$$

Then

$$
F(1)=-\frac{\beta-\gamma}{8 \beta \gamma} \sqrt{\Delta}[(1+m) \sqrt{\beta}-\sqrt{\Delta}]^{3}<0
$$

and

$$
F(-1)=4+\frac{(1+m) \sqrt{\beta}-\sqrt{\Delta}}{2 \beta^{\frac{3}{2}} \gamma} H-\frac{\beta-\gamma}{8 \beta \gamma} \sqrt{\Delta}[(1+m) \sqrt{\beta}-\sqrt{\Delta}]^{3} .
$$

Therefore, by (iii) of Lemma 2.1, we get the desired results (a)-(c).

Theorem 2.5 Let $\beta>\gamma$ and $\Delta>0$, then the following conclusions hold.

(1) If $0<\frac{(1+m) \sqrt{\beta}+\sqrt{\Delta}}{2 \beta^{\frac{3}{2}} \gamma} L-\frac{\beta-\gamma}{8 \beta \gamma} \sqrt{\Delta}[(1+m) \sqrt{\beta}+\sqrt{\Delta}]^{3}<4$, then the point $\mathbf{p}$ is a sink;

(2) if $\frac{(1+m) \sqrt{\beta}+\sqrt{\Delta}}{2 \beta^{\frac{3}{2}} \gamma} L-\frac{\beta-\gamma}{8 \beta \gamma} \sqrt{\Delta}[(1+m) \sqrt{\beta}+\sqrt{\Delta}]^{3}=4$, then the point $\mathbf{p}$ is non-hyperbolic;

(3) if $\frac{(1+m) \sqrt{\beta}+\sqrt{\Delta}}{2 \beta^{3} \gamma} L-\frac{\beta-\gamma}{8 \beta \gamma} \sqrt{\Delta}[(1+m) \sqrt{\beta}+\sqrt{\Delta}]^{3}>4$, then the point $\mathbf{p}$ is a saddle;

(4) if $\frac{(1+m) \sqrt{\beta}+\sqrt{\Delta}}{2 \beta^{\frac{3}{2}} \gamma} L-\frac{\beta-\gamma}{8 \beta \gamma} \sqrt{\Delta}[(1+m) \sqrt{\beta}+\sqrt{\Delta}]^{3}<0$, then the point $\mathbf{p}$ is a source;

(5) if $\frac{(1+m) \sqrt{\beta}+\sqrt{\Delta}}{2 \beta^{\frac{3}{2}} \gamma} L=\frac{\beta-\gamma}{8 \beta \gamma} \sqrt{\Delta}[(1+m) \sqrt{\beta}+\sqrt{\Delta}]^{3}<4$, then the point $\mathbf{p}$ is non-hyperbolic.

Proof The Jacobian matrix $J$ of system (1.5) at the equilibrium point $\mathbf{p}\left(x_{2}, y_{2}\right)$ is

$$
J_{\mathbf{p}}=\left(\begin{array}{cc}
1+x_{2}\left[\alpha\left(1-\frac{\gamma}{\beta}\right)-\frac{\sqrt{\beta \Delta}}{\gamma} x_{2}\right] & -\frac{\alpha \gamma}{\beta} x_{2} \\
\frac{(\beta-\gamma)^{2}}{\gamma} x_{1} & 1-(\beta-\gamma) x_{2}
\end{array}\right) .
$$


Its characteristic equation is

$$
F(\lambda)=\lambda^{2}-\operatorname{Tr}\left(J_{\mathbf{p}}\right) \lambda+\operatorname{Det}\left(J_{\mathbf{p}}\right)=0,
$$

where

$$
\operatorname{Tr}\left(J_{\mathbf{p}}\right)=2-\frac{(1+m) \sqrt{\beta}+\sqrt{\Delta}}{4 \beta^{\frac{3}{2}} \gamma} L
$$

and

$$
\operatorname{Det}\left(J_{\mathbf{p}}\right)=1-\frac{(1+m) \sqrt{\beta}+\sqrt{\Delta}}{4 \beta^{\frac{3}{2}} \gamma} L+\frac{\beta-\gamma}{8 \beta \gamma} \sqrt{\Delta}[(1+m) \sqrt{\beta}+\sqrt{\Delta}]^{3} .
$$

By (2.10) and (2.11), we have

$$
F(1)=\frac{\beta-\gamma}{8 \beta \gamma} \sqrt{\Delta}[(1+m) \sqrt{\beta}+\sqrt{\Delta}]^{3}>0
$$

and

$$
F(-1)=4-\frac{(1+m) \sqrt{\beta}+\sqrt{\Delta}}{2 \beta^{\frac{3}{2}} \gamma} L+\frac{\beta-\gamma}{8 \beta \gamma} \sqrt{\Delta}[(1+m) \sqrt{\beta}+\sqrt{\Delta}]^{3} .
$$

Part (1). If $0<\frac{(1+m) \sqrt{\beta}+\sqrt{\Delta}}{2 \beta^{\frac{3}{2}} \gamma} L-\frac{\beta-\gamma}{8 \beta \gamma} \sqrt{\Delta}[(1+m) \sqrt{\beta}+\sqrt{\Delta}]^{3}<4$, then $F(-1)>0$ and $\operatorname{Det}\left(J_{\mathbf{p}}\right)<1$. By (i.1) of Lemma 2.1, we know that two eigenvalues of (2.9) satisfy $\left|\lambda_{1}\right|<1$ and $\left|\lambda_{2}\right|<1$. Hence, the equilibrium point $\mathbf{p}$ is a sink.

Part (2). If $\frac{(1+m) \sqrt{\beta}+\sqrt{\Delta}}{2 \beta^{\frac{3}{2}} \gamma} L-\frac{\beta-\gamma}{8 \beta \gamma} \sqrt{\Delta}[(1+m) \sqrt{\beta}+\sqrt{\Delta}]^{3}=4$, then $F(-1)=0$. Notice $\operatorname{Tr}\left(J_{\mathbf{p}}\right) \neq$ -2 . So, by (i.2) of Lemma 2.1, we know that two eigenvalues of (2.9) satisfy $\lambda_{1}=-1$ and $\lambda_{2} \neq-1$. Hence, the equilibrium point $\mathbf{p}$ is non-hyperbolic.

Part (3). If $\frac{(1+m) \sqrt{\beta}+\sqrt{\Delta}}{2 \beta^{\frac{3}{2}} \gamma} L-\frac{\beta-\gamma}{8 \beta \gamma} \sqrt{\Delta}[(1+m) \sqrt{\beta}+\sqrt{\Delta}]^{3}>4$, then $F(-1)<0$. By (i.3) of Lemma 2.1, it is easy to see that two eigenvalues of (2.9) satisfy $\left|\lambda_{1}\right|<1$ and $\left|\lambda_{2}\right|>1$. Therefore, the equilibrium point $\mathbf{p}$ is a saddle.

Part (4). If $\frac{(1+m) \sqrt{\beta}+\sqrt{\Delta}}{2 \beta^{\frac{3}{2}} \gamma} L-\frac{\beta-\gamma}{8 \beta \gamma} \sqrt{\Delta}[(1+m) \sqrt{\beta}+\sqrt{\Delta}]^{3}<0$, then $F(-1)>0$ and $\operatorname{Det}\left(J_{\mathbf{p}}\right)>1$. By (i.4) of Lemma 2.1, two eigenvalues of (2.9) satisfy $\left|\lambda_{1}\right|>1$ and $\left|\lambda_{2}\right|>1$. Hence, the equilibrium point $\mathbf{p}$ is a source.

Part (5). If $\frac{(1+m) \sqrt{\beta}+\sqrt{\Delta}}{2 \beta^{\frac{3}{2}} \gamma} L=\frac{\beta-\gamma}{8 \beta \gamma} \sqrt{\Delta}[(1+m) \sqrt{\beta}+\sqrt{\Delta}]^{3}<4$, then $\left|\operatorname{Tr}\left(J_{\mathbf{p}}\right)\right|<2$ and $\operatorname{Det}\left(J_{\mathbf{p}}\right)=1$. By (i.5) of Lemma 2.1, two eigenvalues of (2.9) satisfy $\left|\lambda_{1}\right|=\left|\lambda_{1}\right|=1$, which are a pair of conjugate complex eigenvalues. Therefore, the equilibrium point $\mathbf{p}$ is nonhyperbolic at this time.

\section{Bifurcation analysis}

In this section we are concerned with the bifurcation problems of system (1.5). It has been shown that for certain parametric conditions some of the equilibrium points may be nonhyperbolic, and hence, system (1.5) may undergo some bifurcations phenomena.

It is easy to see from Theorem 2.2 that the stability of the equilibrium point $E_{1}(1,0)$ changes when $\beta$ varies near $\beta_{0}=\gamma$, while the relation in $\alpha, m$ and $\gamma$ is fixed. So, this system 
probably undergoes a bifurcation nearby $\beta_{0}=\gamma$. We are now in a position to consider this problem: what kind of bifurcation is it? For the reader's convenience, we formulate this process step by step.

The first step. Giving a perturbation $\beta_{*}$ of the parameter $\beta=\beta_{0} \triangleq \gamma$, we consider a perturbation of system (1.5) as follows:

$$
\left\{\begin{array}{l}
x(n+1)=x(n) \exp [(1-x(n))(x(n)-m)(x(n)+y(n))-\alpha y(n)], \\
y(n+1)=y(n) \exp \left[\left(\beta_{0}+\beta_{*}\right) x(n)-\gamma(x(n)+y(n))\right],
\end{array}\right.
$$

where $\left|\beta_{*}\right| \ll 1$.

The second step. Let $u(n)=x(n)-1$ and $v(n)=y(n)-0$, which transforms equilibrium point $E_{1}(1,0)$ to the origin $O(0,0)$ and system (3.1) into

$$
\left\{\begin{array}{l}
u \rightarrow(u+1) \exp [-u(u+1-m)(u+v+1)-\alpha v]-1, \\
v \rightarrow v \exp \left[\left(\beta_{0}+\beta_{*}\right)(u+1)-\gamma(u+v+1)\right] .
\end{array}\right.
$$

The third step. Expanding (3.2) as a Taylor series at $\left(u, v, \beta_{*}\right)=(0,0,0)$ up to terms of order 3 produces the following model:

$$
\left\{\begin{aligned}
u(n+1) & =e_{100} u(n)+e_{010} v(n)+e_{200} u^{2}(n)+e_{110} u(n) v(n) \\
& +e_{020} v^{2}(n)+e_{300} u^{3}(n)+e_{210} u^{2}(n) v(n) \\
& +e_{120} u(n) v^{2}(n)+e_{030} v^{3}(n)+O\left(r^{4}\right), \\
v(n+ & 1) \\
= & f_{100} u(n)+f_{010} v(n)+f_{001} \beta_{*}+f_{200} u^{2}(n)+f_{020} v^{2}(n) \\
& +f_{002} \beta_{*}^{2}+f_{110} u(n) v(n)+f_{101} u(n) \beta_{*}+f_{011} v(n) \beta_{*} \\
& +f_{300} u^{3}(n)+f_{030} v^{3}(n)+f_{003} \beta_{*}^{3}+f_{210} u^{2}(n) v(n) \\
& +f_{120} u(n) v^{2}(n)+f_{021} v^{2}(n) \beta_{*}+f_{201} u^{2}(n) \beta_{*}+f_{102} u(n) \beta_{*}^{2} \\
& +f_{012} v(n) \beta_{*}^{2}+f_{111} u(n) v(n) \beta_{*}+O\left(r^{4}\right),
\end{aligned}\right.
$$

where

$$
\begin{aligned}
& r=\sqrt{u^{2}(n)+v^{2}(n)+\beta_{*}^{2}}, \\
& e_{100}=m, \quad e_{010}=-\alpha, \\
& e_{200}=\frac{1}{2}\left(m^{2}+2 m-5\right), \quad e_{020}=\frac{1}{2} \alpha^{2}, \quad e_{110}=-m \alpha+m-1, \\
& e_{210}=\frac{1}{2}\left[\left(5-m^{2}-2 m\right) \alpha+2\left(m^{2}-m-1\right)\right], \quad e_{300}=\frac{1}{6}\left(m^{3}+6 m^{2}-15 m-4\right), \\
& e_{030}=-\frac{1}{6} \alpha^{3}, \quad e_{120}=\frac{1}{2} m \alpha^{2}+\alpha(1-m), \\
& f_{100}=0, \quad f_{010}=1, \quad f_{001}=0, \quad f_{200}=0, \\
& f_{020}=-\gamma, \quad f_{002}=0, \quad f_{110}=0, \quad f_{101}=0,
\end{aligned}
$$




$$
\begin{array}{llll}
f_{011}=1, & f_{300}=0, & f_{030}=\frac{1}{2} \gamma^{2}, & f_{003}=0, \\
f_{210}=0, & f_{120}=0, & f_{021}=-\gamma, & f_{201}=0, \\
f_{102}=0, & f_{012}=\frac{1}{2}, & f_{111}=1 . &
\end{array}
$$

Let

$$
J\left(E_{1}\right)=\left(\begin{array}{cc}
e_{100} & e_{010} \\
f_{100} & f_{010}
\end{array}\right), \quad \text { namely, } \quad J\left(E_{1}\right)=\left(\begin{array}{cc}
m & -\alpha \\
0 & 1
\end{array}\right) .
$$

By some computations, we obtain the eigenvalues and corresponding eigenvectors of ma$\operatorname{trix} J_{E_{1}}$, which are

$$
\mu_{1}=m, \quad \mu_{2}=1,
$$

and

$$
\left(u_{1}, v_{1}\right)^{T}=(1,0)^{T}, \quad\left(u_{2}, v_{2}\right)^{T}=\left(\frac{\alpha}{m-1}, 1\right)^{T},
$$

respectively.

The fourth step. Let the matrix

$$
B=\left(\begin{array}{ll}
u_{1} & u_{2} \\
v_{1} & v_{2}
\end{array}\right), \quad \text { namely } \quad B=\left(\begin{array}{cc}
1 & \frac{\alpha}{m-1} \\
0 & 1
\end{array}\right) .
$$

Denote $s \triangleq \frac{\alpha}{m-1}$. Then $B$ is invertible with

$$
B^{-1}=\left(\begin{array}{cc}
1 & -s \\
0 & 1
\end{array}\right)
$$

Using the transformation below,

$$
(u(n), v(n))^{T}=B(X(n), Y(n))^{T},
$$

we can transform system (3.3) into the following form:

$$
\left\{\begin{array}{l}
X(n+1)=m X(n)+F\left(X(n), Y(n), \beta_{*}\right)+O\left(r^{4}\right) \\
Y(n+1)=Y(n)+G\left(X(n), Y(n), \beta_{*}\right)+O\left(r^{4}\right)
\end{array}\right.
$$

where $r=\sqrt{X^{2}(n)+Y^{2}(n)+\beta_{*}^{2}}$,

$$
\begin{aligned}
& F\left(X(n), Y(n), \beta_{*}\right) \\
& \quad g_{200} X^{2}(n)+g_{020} Y^{2}(n)+g_{002} \beta_{*}^{2}+g_{110} X(n) Y(n)+g_{101} X(n) \beta_{*} \\
& \quad+g_{011} Y(n) \beta_{*}+g_{300} X^{3}(n)+g_{030} Y^{3}(n)+g_{003} \beta_{*}^{3}+g_{210} X^{2}(n) Y(n)
\end{aligned}
$$




$$
\begin{aligned}
& +g_{120} X(n) Y^{2}(n)+g_{201} X^{2}(n) r_{*}+g_{102} X(n) \beta_{*}^{2}+g_{021} Y^{2}(n) \beta_{*} \\
& +g_{012} Y(n) \beta_{*}^{2}+g_{111} X(n) Y(n) \beta_{*}, \\
& G\left(X(n), Y(n), \beta_{*}\right) \\
& =h_{200} X^{2}(n)+h_{020} Y^{2}(n)+h_{002} \beta_{*}^{2}+h_{110} X(n) Y(n)+h_{101} X(n) \beta_{*} \\
& +h_{011} Y(n) \beta_{*}+h_{300} X^{3}(n)+h_{030} Y^{3}(n)+h_{003} \beta_{*}^{3}+h_{210} X^{2}(n) Y(n) \\
& +h_{120} X(n) Y^{2}(n)+h_{201} X^{2}(n) \beta_{*}+h_{102} X(n) \beta_{*}^{2}+h_{021} Y^{2}(n) \beta_{*} \\
& +h_{012} Y(n) \beta_{*}^{2}+h_{111} X(n) Y(n) \beta_{*} \text {, } \\
& g_{200}=\frac{1}{2}\left(m^{2}+2 m-5\right), \quad g_{020}=e_{200} s^{2}+e_{020}-s f_{020}+s e_{110}, \\
& g_{101}=0, \quad g_{011}=-s, \\
& g_{110}=2 s e_{200}+e_{110}, \quad g_{030}=e_{030}-s f_{030}+s^{2} e_{210}+s e_{120}+s^{3} e_{300}, \quad g_{002}=0, \\
& g_{300}=\frac{1}{6}\left(m^{3}+6 m^{2}-15 m-4\right), \quad g_{003}=0, \quad g_{210}=3 s e_{300}+e_{210}, \quad g_{102}=0, \\
& g_{120}=3 s^{2} e_{300}+2 s e_{210}+e_{120}, \quad g_{012}=-\frac{1}{2} s, \quad g_{201}=0, \quad g_{021}=s \gamma-s^{2}, \\
& g_{111}=-s ; \quad h_{200}=0, \quad h_{020}=-\gamma, \quad h_{002}=0, \quad h_{110}=0, \quad h_{101}=0, \\
& h_{011}=1, \quad h_{300}=0, \quad h_{030}=\frac{1}{2} \gamma^{2}, \quad h_{003}=0 \text {, } \\
& h_{210}=0, \quad h_{120}=0, \quad h_{201}=0, \quad h_{012}=\frac{1}{2} \text {, } \\
& h_{021}=s-\gamma, \quad h_{102}=0, \quad h_{111}=1 .
\end{aligned}
$$

The fifth step. Determine the center manifold $W^{c}(0,0)$ of system (3.4) at the equilibrium point $O(0,0)$ in a small neighborhood of $\beta_{*}=0$. By the center manifold theorem, we can assume that the approximate representation of the central manifold $W^{c}(0,0)$ is as follows:

$$
\begin{aligned}
W^{c}(0,0)=\{ & (X, Y): X=\ell_{10} Y+\ell_{01} \beta_{*}+\ell_{20} Y^{2}+\ell_{11} Y \beta_{*}+\ell_{02} \beta_{*}^{2} \\
& \left.+\ell_{30} Y^{3}+\ell_{21} Y^{2} \beta_{*}+\ell_{12} Y \beta_{*}^{2}+\ell_{03} \beta_{*}^{3}+O\left(r^{4}\right)\right\},
\end{aligned}
$$

where $r=\sqrt{Y^{2}+\beta_{*}^{2}}$ and the coefficients $\ell_{i j}, i, j=0,1,2,3$ are to be defined. Through some computations, one may get

$$
\begin{aligned}
\ell_{10}= & 0, \quad \ell_{01}=0, \quad \ell_{20}=\frac{s^{2} e_{200}+e_{020}+s e_{110}-s f_{020}}{1-m}, \quad \ell_{02}=0, \\
\ell_{11}= & -\frac{s f_{011}}{1-e_{100}}=\frac{\alpha}{(1-m)^{2}}, \quad \ell_{12}=-\frac{s f_{012}+\ell_{11} f_{011}}{1-e_{100}}=-\frac{(m+1) \alpha}{2(1-m)^{3}}, \\
\ell_{30}= & \frac{1}{(1-m)^{2}}\left(2 s e_{200}+e_{110}-s f_{021}\right)\left(s^{2} e_{200}+e_{020}+s e_{110}-s f_{020}\right) \\
& +\frac{1}{1-m}\left(s^{3} e_{300}+e_{030}+s^{2} e_{210}+s e_{120}-s f_{030}\right), \quad \ell_{03}=0, \\
\ell_{21}= & \frac{1}{m-1}\left(s f_{021}+s^{2} f_{111}+2 \ell_{20} f_{011}+\ell_{11} f_{021}\right)+\frac{1}{1-m}\left(2 s e_{200}+e_{110}-f_{021}\right) \ell_{11} .
\end{aligned}
$$


Hence, when $\beta_{*}=0$, the center manifold reads

$$
W^{c}(0,0)=\left\{(X, Y): X=\ell_{20} Y^{2}+\ell_{30} Y^{3}+O\left(Y^{4}\right)\right\} .
$$

Therefore, system (3.4) restricted to the center manifold $W^{c}(0,0)$ has the expression

$$
Y \rightarrow Y-\gamma Y^{2}+\frac{1}{2} \gamma^{2} Y^{3}+O\left(Y^{4}\right)
$$

Combining the above analysis and the conclusion in [17], we obtain the second main result below.

Theorem 3.1 Consider system (1.5). Assume that $\beta=\gamma$ and the other parameters in system (1.5) are fixed. Give a perturbation $\beta_{*}$ of the parameter $\beta=\beta_{0}:=\gamma$. Then system (1.5) undergoes a fold bifurcation at equilibrium point $E_{1}(1,0)$ when the parameter $\beta_{*}$ varies in the small neighborhood of the origin. Moreover, the closed orbit bifurcating from $E_{1}(1,0)$ is unstable.

\section{Numerical simulation}

In this section, we give some numerical simulation results to illustrate the theoretical analysis obtained in the previous sections, and show some new interesting complex dynamics of system (1.5). To do this, the initial value is taken to be $(1.0,0.5)$ and 1000 iteration steps are implemented for each simulation.

On the basis of Theorem 2.1, we know that system (1.5) has three boundary nonnegative equilibrium points $E_{0}(0,0), E_{1}(1,0)$ and $E_{m}(m, 0)$. For $E_{1}(1,0)$, its two eigenvalues are $\lambda_{1}=$ $m<1$ and $\lambda_{2}=\exp (\beta-\gamma)$.

Vary $\beta$ in the range $5 \leq \beta \leq 20$, and fix $m=0.1, \alpha=1, \gamma=10$. The bifurcation diagram is plotted in Fig. 1(a)-(d). We see that the equilibrium point $E_{1}(1,0)$ is stable for $5<\beta<12$, and loses its stability at the fold bifurcation parameter value $\beta=12$. Moreover, a chaotic set emerges with the increasing of $\beta$. But, from Fig. 1(b) and (d), we know that when $\beta$ increases to some fixed value the prey (predator) becomes extinct.

The maximum Lyapunov exponents corresponding to Fig. 1 are calculated and plotted in Fig. 2.

The phase portraits are plotted in Fig. 3. From Fig. 3(a)-(f), we can see the predator is extinct. Predator and prey are extinct from Fig. 3(f). Figures 1-3 illustrate that Theorem 3.1 is correct.

\section{Result and discussion}

In this paper, we have considered a discrete predator-prey system with strong Allee effect on the prey and ratio-dependent functional response. After a counterexample is formulated to demonstrate the limitations and errors for the local stability of the equilibrium point $\mathbf{p}$ stated in Theorem 4.2 [1], a complete determining criterion is presented for the local stability of these equilibria in Theorem 1.1. What is more important is that the stability and bifurcation of the equilibrium point $E_{1}(1,0)$ of this system are stated. The results obtained indicate that the stability of this system is influenced by many factors: the death rate of the predator, the prey death rate, the predator growing ability and the Allee threshold. Especially, the predator growing ability $\beta$ varies around the death rate $\gamma$ of the 


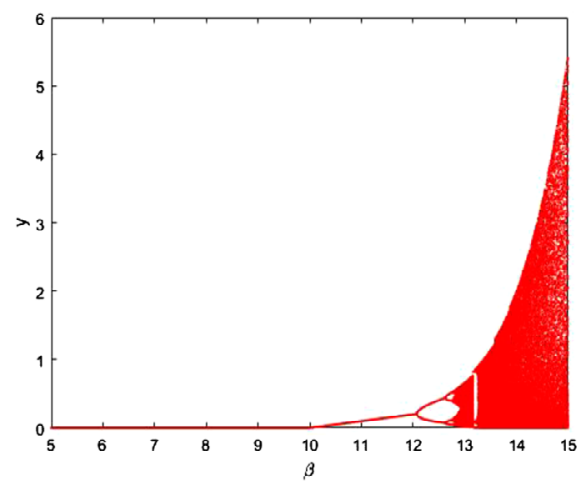

(a) $\beta \in[5,15]$

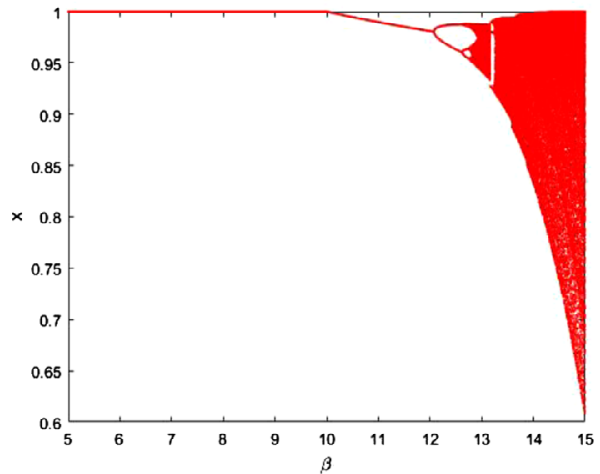

(c) $\beta \in[5,15]$

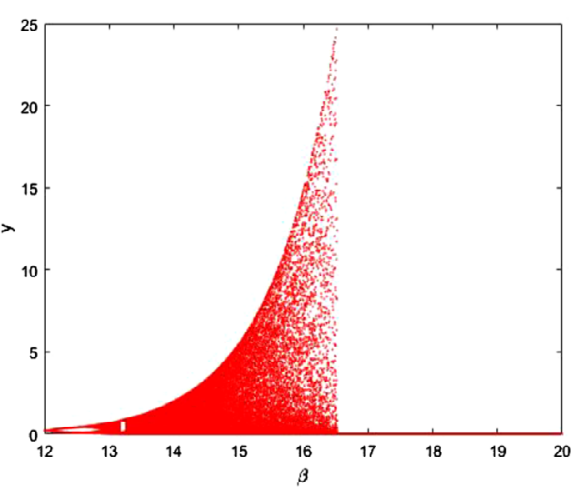

(b) $\beta \in[12,20]$

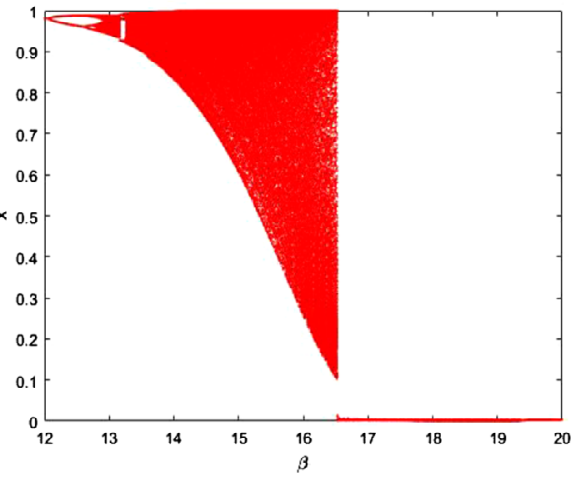

(d) $\beta \in[12,20]$

Figure 1 The dynamics of $x$ and $y$

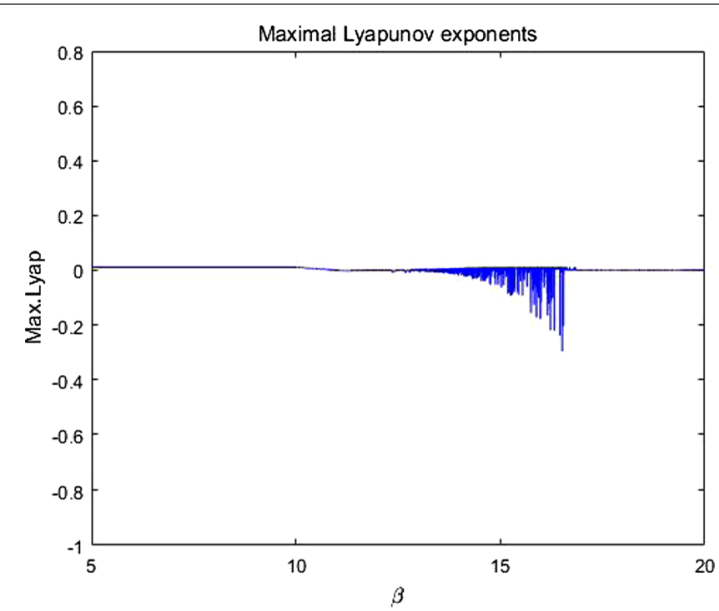

Figure 2 Maximum Lyapunov exponents corresponding to Fig. 1

predator, the fold bifurcation will occur at this system. Some more interesting dynamical properties, such as chaos, are also obtained by a numerical simulation, which shows that the system is worthy of further theoretical research.

Especially, the extinction and coexistence of both populations have also been found to occur for some fixed parameter sets. This sufficiently demonstrates that the dynamical 


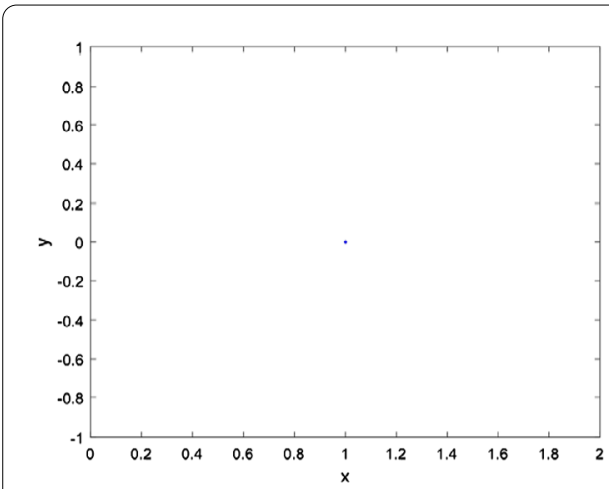

(a) $\beta=9$

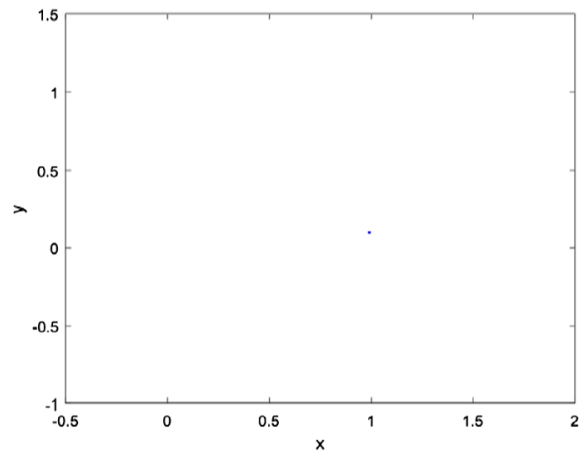

(c) $\beta=11$

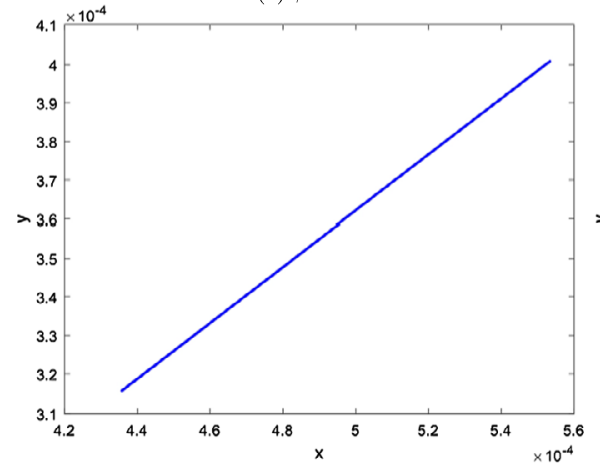

(e) $\beta=17$

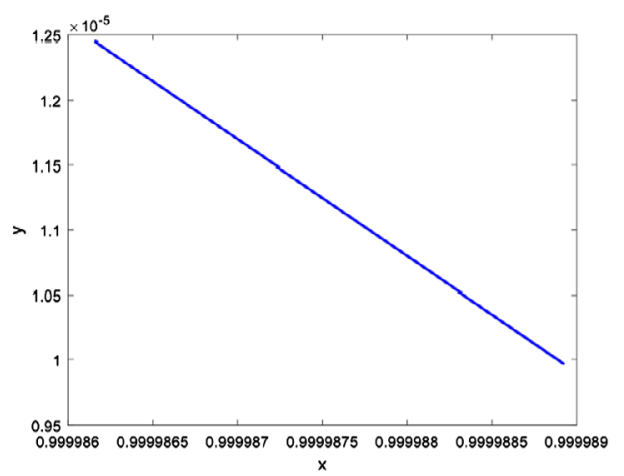

(b) $\beta=10$

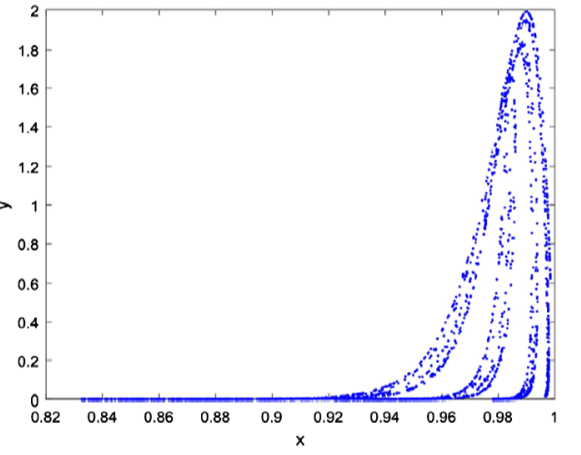

(d) $\beta=14$

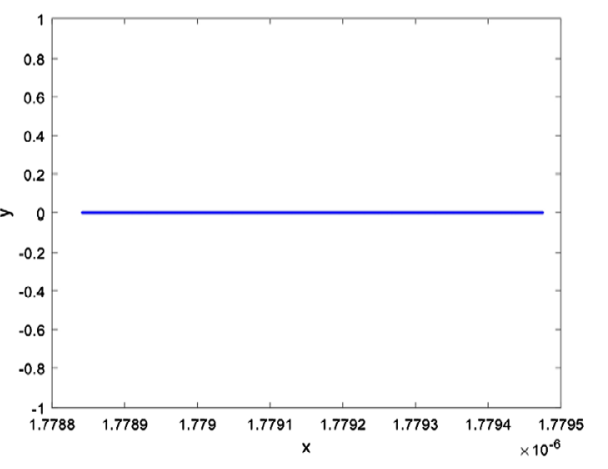

(f) $\beta=19$

Figure 3 Phase portrait of the system (1.5) versus $\beta$

properties of this system may be highly sensitive to the living environment. It deserves further consideration as regards the Allee effect and the interaction of a ratio-related predator on the living environment of the populations.

It should be pointed out that there are differences for the obtained results between the systems (1.1) and (1.5). Because the system (1.1) is a continuous-time model, while the system (1.5) is a discrete model, i.e., a discrete version of the system (1.1), it is reasonable that there exist differences as regards the derived conclusions between them. In fact, the results obtained by the authors in [1] demonstrate that the system (1.1) exhibits the B-T bifurcation, limit cycles, homoclinic orbits and heteroclinic connections. But the results obtained by us in this paper display that there is a flip bifurcation in the system (1.5), which does not occur in the system (1.1). This indicates that some properties of discrete model sometimes cannot be obtained by the discretization of the corresponding contin- 
uous model. This further implies that it is meaningful to study the discretization of the continuous model.

\author{
Acknowledgements \\ The authors sincerely thank the reviewers for their valuable suggestions and useful comments that have led to the \\ present improved version.
}

\title{
Funding
}

This work of the first author is supported by Natural Science Foundation of China under grant No. 11701513 and No. 11426203. This work of the second author is supported by Natural Science Foundation of China under grant No. 61473340 and Natural Science Foundation of Zhejiang University of Science and Technology under grant No. F701108G14.

\section{Competing interests}

The authors declare that they have no competing interests.

\section{Authors' contributions}

All authors contributed equally and significantly in writing this paper. All authors have read and approved of the final manuscript.

\section{Publisher's Note}

Springer Nature remains neutral with regard to jurisdictional claims in published maps and institutional affiliations.

Received: 11 April 2018 Accepted: 27 August 2018 Published online: 12 September 2018

\section{References}

1. Aguirre, P., Flores, D., González-Olivares, E.: Bifurcations and global dynamics in a predator-prey model with a strong Allee effect on the prey, and a ratio-dependent functional response. Nonlinear Anal., Real World Appl. 16, 235-249 (2014)

2. Aguirre, P., González-Olivares, E., Torres, S.: Stochastic predator-prey model with Allee effect. Nonlinear Anal., Real World Appl. 14(1), 768-779 (2013). https://doi.org/10.1016/j.nonrwa.2012.07.032

3. Arditi, R., Saiah, H.: Empirical evidence of the role of heterogeneity in ratio-dependent consumption. Ecology 73 , 1544-1551 (1992)

4. Arditi, R., Ginzburg, L.: Coupling in predator-prey dynamics: ratio-dependance. J. Theor. Biol. 139, $311-326$ (1989)

5. Berec, L., Angulo, E., Counchamp, F.: Multiple Allee effects and population management. Ecol. Model. 22, 185-191 (2006)

6. Chen, B., Chen, J.: Bifurcation and chaotic behavior of a discrete singular biogical economic system. Appl. Math. Comput. 219(5), 2371-2386 (2012)

7. Chicone, C.: In: Ordinary Differential Equations with Applications, 2nd edn. Texts in Applied Mathematics, vol. 34. Springer, Berlin (2006)

8. Courchamp, F., Berec, L., Gascoigne, J.: Allee Effects in Ecology and Conservation. Oxford University Press, London (2008)

9. Gause, G.: The Struggle for Existence. Williams \& Wilkins, Baltimore (1934)

10. Gascoigne, J., Lipcius, R.: Allee effects in marine systems. Mar. Ecol. Prog. Ser. 269, 49-59 (2004)

11. Haque, M.: Ratio-dependent predator-prey models of interacting populations. Bull. Math. Biol. 71, 430-452 (2009)

12. Han, W., Liu, M.: Stability and bifurcation analysis for a discrete-time model of Lotka-Volterra type with delay. Appl. Math. Comput. 217(12), 5449-5457 (2011)

13. He, Z., Lai, X.: Bifurcation and chaotic behavior of a discrete-time predator-prey system. Nonlinear Anal., Real World Appl. 12(1), 403-417 (2011)

14. Hu, Z., Teng, Z., Zhang, L.: Stability and bifurcation analysis of a discrete predator-prey model with nonmonotonic functional response. Nonlinear Anal., Real World Appl. 12(4), 2356-2377 (2011)

15. Jana, D.: Chaotic dynamics of a discrete predator-prey system with prey refuge. Appl. Math. Model. 224, $848-865$ (2013)

16. Ghaziani, R.K., Govaerts, W., Sonck, C.: Resonance and bifurcation in a discrete-time predator-prey system with Holling functional response. Nonlinear Anal., Real World Appl. 13(3), 1451-1465 (2012). https://doi.org/10.1016/j.nonrwa.2011.11.009

17. Kuzenetsov, Y.: Elements of Applied Bifurcation Theory, 3rd edn. Applied Mathematical Sciences, vol. 112. Springer, New York (2004). https://doi.org/10.1007/978-1-4757-3978-7

18. Lu, H., Wang, W.: Dynamics of a delayed discrete semi-ratio-dependent predator-prey system with Holling type IV functional response. Adv. Differ. Equ. 2011, 7 (2011)

19. Li, X.: Bifurcation analysis of a predator-prey system with sex-structure and sexual favoritism. Adv. Differ. Equ. 2013, 219 (2013). https://doi.org/10.1186/1687-1847-2013-219

20. Misra, O., Sinha, P., Singh, C.: Stability and bifurcation analysis of a prey-predator model with age based predation. Appl. Math. Model. 37(9), 6519-6529 (2013). https://doi.org/10.1016/j.apm.2013.01.036

21. Robinson, C.: Dynamical Systems: Stability, Symbolic Dynamics, and Chaos. CRC Press, London (1995)

22. Ruan, S., Xiao, D.: Global analysis in a predator-prey system with nonmononotonic functional response. SIAM J. Appl. Math. 61(4), 1445-1472 (2001)

23. Turchin, P.: Complex Population Dynamics. A Theoretical/Empirical Synthesis. Monographs in Population Biology, vol. 35. Princeton University Press, Princeton (2003) 
24. Wang, C., Li, X.: Further investigations into the stability and bifurcation of a discrete predator-prey model. J. Math. Anal. Appl. 422, 920-939 (2015)

25. Xiao, D., Ruan, S.: Global dynamics of ratio-dependent predator-prey system. J. Math. Biol. 43(3), 268-290 (2001). https://doi.org/10.1007/s002850100097

Submit your manuscript to a SpringerOpen ${ }^{\circ}$ journal and benefit from:

- Convenient online submission

$\checkmark$ Rigorous peer review

- Open access: articles freely available online

- High visibility within the field

- Retaining the copyright to your article

Submit your next manuscript at $\gg$ springeropen.com 